\title{
Feasibility studies of X-ray computed tomography for forensic examination of single fibers
}

\author{
Wataru TADANO,****, Eishi TANABE, *** Jens R. STELLHORN,**** \\ Kenji KOMAGUCHI,**** Masaru TAKEUCHI,* and Shinjiro \\ HAYAKAWA $* * * *, \dagger$
}

* Forensic Science Laboratory, Hiroshima Prefectural Police H.Q., 2-26-3 Konan, Naka, Hiroshima, 730-0825, Japan

** Department of Applied Chemistry, Graduate School of Engineering, Hiroshima University, 1-4-1 Kagamiyama, Higashi-Hiroshima, 739-8527, Japan

*** West Region Industrial Research Center, Hiroshima Prefectural Technology Research Institute, 3-13-26 Kagamiyama, Higashi-Hiroshima, 739-0046, Japan **** Department of Applied Chemistry, Graduate School of Advanced Science and Engineering, Hiroshima University, 1-4-1 Kagamiyama, Higashi-Hiroshima, 739-8527, Japan

\footnotetext{
†E-mail: $\underline{\text { d172104@hiroshima-u.ac.jp }}$

†E-mail: hayakawa@hiroshima-u.ac.jp
} 


\begin{abstract}
Non-destructive observations of cross-sectional shapes of acrylic single fibers using Xray computed tomography (CT) were performed. A commercial X-ray CT apparatus (Rigaku nano3DX) was employed because of its micrometer scale spatial resolution and remarkable image contrast for soft matters. We assessed two types of sample support, a paraffin strip and a nylon string, for single fiber samples in terms of easy handling and sample recovery. The fixed individual single fibers were loaded into a narrow polyimide tube in both cases, and the tube ensured that the sample remained in the field of view during the CT measurement. In both cases, the cross-sectional shapes of individual single fibers could be distinguished, with a circular shape for one sample and a triangular shape for the other. However, the support using a nylon string was found to be more suitable for the further analysis. The cross sectional profile of the obtained tomographic image showed clear difference between polyimide and nylon. The intensity ratio or the image contrast corresponded to that of the local densities. It was also found that the effect of the artifact appeared around the boundaries of the objects, but the local density can still be utilized for the examination of the individual single fibers.
\end{abstract}




\section{Introduction}

Single fibers are typical forensic trace evidence left unconsciously at crime scenes. For classes of synthetic fibers, polyester, nylon, and acrylic are dominate the market. The analytical importance of forensic trace evidence is widely known as Locard's exchange principle. ${ }^{1-3}$ In forensic examination, a requested sample is compared with a reference sample based on chemical or physical properties, and non-destructive methods are important. The sample should be conserved for the further analysis. For the analysis of individual fibers, non-destructive methods such as microscopic Fourier transform infrared (FT-IR) spectroscopy, microscopic Raman spectroscopy and other microspectroscopies are widely used in forensic science laboratories in Japan. For studies done before 2019 on fiber analysis, the reader is referred to reviews by Farah et al., ${ }^{4}$ Meleiro and García-Ruiz, ${ }^{5}$ Śmigiel-Kamińska et al., ${ }^{6}$ Mistek et al., ${ }^{7}$ and $\mathrm{Hu}$ et al. ${ }^{8}$ In recent years, various studies on the forensic examination of single fibers have been published. Peets et al. employed reflectance FT-IR (r-FT-IR) spectroscopy as an alternative to the conventional method using attenuated total reflectance FT-IR (ATRFT-IR) spectroscopy. ${ }^{9}$ The main drawback of ATR-FT-IR is the need to apply significant pressure to the textile, whereas r-FT-IR is the contactless non-invasive technique. r-FTIR was demonstrated to be more successful than ATR-FT-IR in the examination between the amide-based fibers wool, silk and polyamide.

Reffner et al. presented a more efficient polarized light microscopy (PLM) to analyze optically anisotropic synthetic fibers. ${ }^{10}$ The traditional PLM method requires the measurement of the fiber's two principal refractive indices and has to be changed until a matching medium is found for each of the fiber's values. The refractive indices of the mounting media they presented had values intermediate to the fiber's two 
principal refractive indices, and provided the differentiation of fiber compositions while minimalizing the number of mounting media.

Nishiwaki et al. performed vertically focused synchrotron radiation microbeam Xray fluorescence (SR- $\mu$-XRF) for the analysis of white polyester clothing fibers. ${ }^{11}$ The white fibers are generally difficult to differentiate with conventional methods, and trace elemental compositions related to the catalysts used during the production was utilized.

Synthetic fibers have various cross-sectional shapes depending on the shape of the nozzle that is used for extrusion such as circular, triangular, star-shaped, heart-shaped, pentagonal, Hollofil (4-hole Fiberfill), and Quallofil (7-hole Fiberfill). Although Kirkbride et al. applied confocal laser scanning microscopy (CLSM) as a fluorescencebased non-destructive method to observe transverse and longitudinal cross-sectional shapes of single fibers, ${ }^{12}$ this method is not introduced in forensic science laboratories in Japan. Productions of cross-sections of fibers are destructive. ${ }^{13}$ The procedure requires skilled technicians, and the observation of cross-sections is often omitted.

In this study, we employed X-ray computed tomography (CT) for the first time as a new non-destructive method to observe cross-sectional shapes of individual fibers. So far, studies on application of X-ray CT in forensic practice such as for gunshot wounds, tool marks on bone, age determination, bone pathology, and post-mortem interval have been published. ${ }^{14}$ Recently the spatial resolution of the commercial X-ray CT devices has reached to the submicron level. ${ }^{15}$ If cross-sectional shapes of single fibers can be observed non-destructively with a resolution of several micrometers to sub-micrometers, it can be utilized as a new method for examination of differences between individual single fibers.

The transmitted X-ray intensity I through a sample thickness of $t$ for a homogeneous object is given as the following equation for the monochromatic X-rays: ${ }^{16}$ 


$$
I=I_{0} \exp (-\mu t)
$$

where $I_{0}$ is the incident intensity. The observed optical density $\mu t$, and the linear absorption coefficient $\mu$ are defined with the product of the mass absorption coefficient $\mu_{\mathrm{m}}$ and the density of the sample $\rho$. When the mass fractions of a sample are given, the $\mu_{\mathrm{m}}$ of the compound is calculated as the weighted sum of the tabulated mass absorption coefficient of the elements in the sample. When the monochromatized or quasimonochromatic X-rays are utilized for CT measurements, the reconstructed CT images provide information on the local X-ray absorption coefficients at each pixel in a crosssectional image, and $t$ is an effective path length. The measured absorption coefficient $\mu$ can be regarded as the value proportional to the density of the organic sample $\rho .{ }^{17}$ Using CLSM, non-destructive observation of cross-sectional shapes of fibers is possible without special mounting techniques such as fluorescent staining. However, using X-ray CT, it may be possible to distinguish not only differences in cross-sectional shapes between individual fibers but also density differences between them. If not only crosssectional shapes but also density differences can be non-destructively and simultaneously be distinguished, high-resolution X-ray CT will be a powerful method for forensic examination.

In this study, aiming to apply X-ray CT as a new method for forensic examination of single fibers, we non-destructively observed and compared acrylic single fibers with known cross-sectional shapes by high-resolution X-ray CT. Furthermore, we also examined the feasibility of examination by density differences of single fibers.

\section{Experimental}




\section{$X$-ray CT measurements and $3 D$ reconstructions}

A commercial X-ray CT apparatus (Rigaku nano3DX) ${ }^{15}$ was used for the measurements. Figure 1 shows the schematic diagram of CT measurements using the nano3DX. The white X-rays from a rotating anode X-ray source are irradiated to a sample as a quasi-parallel beam, and a transmitted X-ray image of the sample is converted to visible light by the scintillator. Compared to the conventional commercial X-ray CT apparatus, the contribution of the characteristic X-rays from a target is enhanced. The image on the scintillator is magnified with the optical lens, and the magnified image is acquired with a complementary metal oxide semiconductor (CMOS) camera. Unlike the conventional cone-beam method, using the nano3DX, X-rays can be regarded as a quasi-parallel beam, and the spatial resolution is determined by the magnification of the lens when the sample-to-scintillator distance XD is small. The spatial resolution and the field of view (FOV) can be adjusted with the lens parameter, and the L1080 lens provided the FOV of $2.67 \times 2.67 \mathrm{~mm}^{2}$ with the calculated spatial resolution of $1.3 \mu \mathrm{m}$ in the pixel size on the CMOS camera. A series of 1000 projections were acquired with the continuous mode. The averaged exposure time was $1 \mathrm{~s}$ for the projection, and it took about 20 minutes for one acquisition. The spatial resolution or voxel size was the same as the pixel size of $1.3 \mu \mathrm{m}$ with the XD of $2 \mathrm{~mm}$. The entire sample was within the FOV during the rotation without blurring.

The advantage of the nano3DX is the utilization of relatively low energy X-rays, which provides a better image contrast for soft matter. In this study, the quasimonochromatic $\mathrm{X}$-rays lines from a $\mathrm{Cu}$ target were utilized, with the $\mathrm{Cu}$ target operating at $40 \mathrm{kV}$ and $30 \mathrm{~mA}$.

A $14 \mathrm{~mm}$ length polyimide tube was mounted on the polyether ether ketone (PEEK) base, and the PEEK base was mounted onto an aluminum rod with a M2 screw. 
The thickness of the tube was $60 \mu \mathrm{m}$ and the inner diameter was $2 \mathrm{~mm}$. A sample was loaded into the tube, and the height of the sample could be adjusted to the X-ray beam. The FOV was selected to be greater than the outer diameter of the tube, and a sample in the tube remained in the FOV during the CT measurement when the tube was properly aligned onto the rotational center. The loaded sample could be easily recovered.

A filtered back projection (FBP) type reconstruction algorithm ${ }^{18}$ was utilized with a commercial software, TomoShop ${ }^{\circledR}$ developed by Midorino Research Co., Ltd. ${ }^{19}$ Misalignment correction was executed in advance of the $3 \mathrm{D}$ reconstruction by the software. The voxel size was $(1.3 \mu \mathrm{m})^{3}$. Sliced images of the reconstructed data were saved in the 8-bit JPEG format.

\section{Single fibers and their fixations}

Two types of acrylic single fibers were obtained from Japan Exlan Co., Ltd. One fiber, K8 had a circular cross-sectional shape, and the thickness was 3 deniers, where 1 denier (d) equals to $1 \mathrm{~g} / 9000 \mathrm{~m}$. The other fiber, F830 had the triangular crosssectional shape, and the thickness was $2.5 \mathrm{~d}$.

For forensic examinations, the recovery of the sample without any significant damage after the examination is extremely important. Furthermore, to maintain a high spatial resolution in the X-ray CT measurements, the sample should be stable during a series of projection measurements. We assessed two types of sample support for single fiber samples. One is a paraffin film of $31 \mu \mathrm{m}$ in thickness. The paraffin film was cut into strips of approximately $1 \mathrm{~mm} \times 14 \mathrm{~mm}$. The edges of a single fiber were mechanically fixed into the strips as shown in Fig. 2(a). The other is a commercial nylon string of $0.17 \mathrm{~mm}$ in diameter. An individual single fiber was fixed to the nylon string of about $14 \mathrm{~mm}$ in length, and one end of the fiber and the string was fixed with a 
piece of scotch tape as shown in Fig. 2(b). To ensure the easier recovery of the sample, the fixation of the one end of the fiber was employed. The calculated transmittance of the polyimide tube, the paraffin film, and the nylon string with $8.04 \mathrm{keV} \mathrm{X}$-rays were $95.1 \%, 96.6 \%$, and $90.3 \%$, respectively. ${ }^{20}$

\section{Results and Discussion}

\section{Observation of cross-sectional images of single fibers}

Figure 3(a) shows the volume rendering (VR) display of the CT image from single fibers fixed to the paraffin films. Figure 3(b) shows an example of the cross-sectional view of Fig. 3(a) that contains both $\mathrm{K} 8$ and F830 fibers. The polyimide tube was accidentally distorted. Fibers appeared to be apart from the paraffin films because only the edges of the individual fibers were fixed. Figure 3(c) and 3(d) show enlarged views for K8 and F830 single fibers in Fig. 3(b), respectively. It can be seen that micrometerscale spatial resolution is obtained, and that the cross-sectional shapes (circular for K8 vs triangular for F830) can be distinguished.

Figure 4(a) shows the VR display of the CT image from single fibers fixed to the nylon strings. Fig. 4(b) shows an example of the cross-sectional view of Fig. 4(a). Again, fibers appeared to be apart from the nylon strings because only one end of the fiber and the string was fixed. Figure 4(c) and 4(d) show enlarged views for K8 and F830 single fibers in Fig. 4(b), respectively. Also in this case, the cross-sectional shapes can be easily distinguished. Additionally, areas of the cross-section of K8 and F830 single fibers were determined. The diameter of $\mathrm{K} 8$ and the length of the triangular crosssection of F830 were $19.5 \mu \mathrm{m}$ and $22.1 \mu \mathrm{m}$ determined from the obtained CT image, 
and the areas were $299 \mu \mathrm{m}^{2}$ and $211 \mu \mathrm{m}^{2}$, respectively. They were converted to 3.2 and 2.3 in denier unit with the use of the density of acrylic (polyacrylonitrile) of 1.184 $\mathrm{g} / \mathrm{cm}^{3}{ }^{21}$ These values showed fairly good correspondence with the reference values of 3 and 2.5 .

Finally, although cross-sectional shapes can be distinguished in both cases, the latter method using the nylon string is relatively easy to fix. Therefore, the chance of losing the sample might be lower for this approach.

\section{Determination of local density}

Figure 5(a) shows the same cross-sectional view as Fig. 4(b), and Fig. 5(b) shows the intensity profile on the line shown in Fig. 5(a). The intensity corresponds to the averaged local linear absorption coefficient $\mu$ in the voxel. The unit of the horizontal axis in Fig. 5(b) is in $\mu \mathrm{m}$. The left and right peaks corresponded to the walls of the polyimide tube, and the central peak corresponds to the nylon string. The thickness of the tube and of the string could be determined from the data precisely. Table 1 shows the net intensity and the standard deviation in Fig. 5(b), the density, ${ }^{21}$ and their ratios. Around the boundary of the tube and the string, negative and positive changes of the intensities were observed. They most probably attributed to the artifacts related to the FBP-method. ${ }^{17,22}$ The back ground (BG) might be attributed to the attenuation caused by the air. Net intensities shown in this table were averaged values over 50 points excluding the artifact around the boundary. Clear difference in intensities was observed between polyimide and nylon. It is indicated that the density difference between polyimide and nylon is the reason for the intensity difference, and the intensity ratio corresponds well to the density ratio.

Figure 6(a) displays the same image as Fig. 5(b), but the intensity profiles (white 
thin dotted line and the white thick dotted line), as shown in Fig. 6(b) now include the single fiber samples. The peaks indicated by the arrows correspond to the K8 and F830 single fibers, respectively. The density of acrylic is almost the same as that of nylon, and is smaller than that of the polyimide. However, the observed intensity was larger than that of the polyimide. Figure 6(c) and 6(d) show enlarged intensity profiles for the F830 and K8 single fibers in Fig. 6(b), respectively. The intensity of the small single fiber might be affected with the artifacts mentioned previously.

\section{Conclusions}

The non-destructive cross-sectional observation of single fibers was demonstrated by using a high-resolution X-ray CT. Two types of fixation methods for single fibers were examined, and the method using the nylon string was determined to be suitable in terms of easy fixation and sample recovery. The cross-sectional shapes of the fibers could be distinguished, with a circular shape for one sample and a triangular shape for the other. It was also found that the density difference between polyimide and nylon could be distinguished as the intensity ratio in the tomographic image. Although effects of the artifacts appeared around the boundaries of the objects, it was demonstrated that the local density might be utilized for the examination of the individual single fibers. The possibility of the determination of the local density is advantageous compared to the CLSM, and the performance of the high-resolution X-ray CT can be powerful tool for forensic investigation.

\section{Acknowledgements}


Some of authors (E. T., K. K. and S. H.) are members of the group, Materials ModelBased Research (MBR) Project in Digital Monozukuri (Manufacturing) Education and Research Center, Hiroshima University. We are grateful for Prof. Joji Ohshita of Hiroshima University for the introduction of the CT instrument as a project leader. We would like to thank Dr. Hirosuke Sumida of Mazda corp. for his technical suggestions and encouragements. The special thanks go to Mr. Tatsuya Asada in the technical center in Hiroshima University for fabricating the sample holder for CT measurements. 


\section{References}

1. E. Locard, Amer. J. Police Sci., 1930, 1, 276.

2. E. Locard and D. J. Larson, Amer. J. Police Sci., 1930, 1, 401.

3. E. Locard, Amer. J. Police Sci., 1930, 1, 496.

4. S. Farah, K. R. Kunduru, T. Tsach, A. Bentolila, and A. J. Domb, Polym. Adv. Technol., 2015, 26, 785.

5. P. P. Meleiro and C. Garciá-Ruiz, Appl. Spectrosc. Rev., 2016, 51, 258.

6. D. Śmigiel-Kamińska, J. Pośpiech, J. Makowska, P. Stepnowski, J. Wąs-Gubała, and J. Kumirska, Molecules, 2019, 24, 613.

7. E. Mistek, M. A. Fikiet, S. R. Khandasammy, and I. K. Lednev, Anal. Chem., 2019, $91,637$.

8. C. Hu, H. Mei, H. Guo, and J. Zhu, Forensic. Chem., 2020, 18, 100221.

9. P. Peets, K. Kaupmees, S. Vahur, and Ivo Leito, Heritage Sci., 2019, 7, 93.

10. J. A. Reffner, B. W. Kammrath, and S. Kaplan, J. Forensic Sci., 2020, 65, 744.

11. Y. Nishiwaki, S. Honda, T. Yamato, R. Kondo, A. Kaneda, and S. Hayakawa, J. Forensic Sci., 2020, 65, 1474.

12. K. P. Kirkbride and S. R. Tridico, Forensic Sci. Int., 2010, 195, 28.

13. J. Robertson, C. Roux, K. G. Wiggins, "Forensic Examination of Fibres", 3rd ed. 2017, CRC Press, FL, 33487-2742, USA.

14. G. N. Rutty, A. Brough, M. J. P. Biggs, C. Robinson, S. D. A. Lawes, and S. V. Hainsworth, Forensic Sci. Int., 2013, 225, 60.

15. Y. Takeda and K. Hamada, Rigaku J., 2015, 31, 10.

16. S. R. Stock, "MicroComputed Tomography: Methodology and Applications", 2nd ed. 2019, CRC Press, FL, 33487-2742, USA.

17. D. H. Phillips and J. J. Lannutti, NDT \& E Int., 1997, 30, 339. 
18. M. Grass, Th. Köhler, and R. Proksa, Phys. Med. Biol., 2000, 45, 329.

19. H. Yang, K. Tomi and H. Kudo, Med. Imaging Technol., 2012, 30, 192 (in Japanese).

20. The Center for X-Ray Optics (CXRO), https://www.cxro.lbl.gov/.

21. Scientific Polymer Products Inc., https://scipoly.com/.

22. H. Qu, F. Xu, X. Lu, H. Miao, T. Xiao, and Z. Zhang, Procedia Eng., 2010, 7, 63. 
Table 1 Net intensities averaged for the central points over 50 points excluding the artifact around the boundary, density of polyimide and nylon, and their ratios.

\begin{tabular}{ccc}
\hline & Averaged net intensity $(n=50)$ & Density $^{21}$ \\
\hline Polyimide & $121 \pm 9$ & $1.40 \mathrm{~g} / \mathrm{cm}^{3}$ \\
Nylon & $93 \pm 7$ & $1.12 \mathrm{~g} / \mathrm{cm}^{3}$ \\
\hline Ratio (Polyimide / Nylon) & $1.3 \pm 0.2$ & 1.25 \\
\hline
\end{tabular}




\section{Figure Captions}

Fig. 1 (a) Schematic diagram of CT measurements using the nano3DX. (b) Enlarged view around the sample.

Fig. 2 Schematic diagram of sample fixations. (a) Single fiber fixed in the cut of the paraffin film. (b) Single fiber fixed to the nylon string.

Fig. 3 (a) VR display of the CT image from single fibers fixed to paraffin films. (b) Example of the cross-sectional view of Fig. 3(a). (c) Enlarged view for the K8 single fiber in Fig. 3(b). (d) Enlarged view for the F830 single fiber in Fig. 3(b).

Fig. 4 (a) VR display of the CT image from single fibers fixed to nylon strings. (b) Example of the cross-sectional view of Fig. 4(a). (c) Enlarged view for the K8 single fiber in Fig. 4(b). (d) Enlarged view for the F830 single fiber in Fig. 4(b).

Fig. 5 (a) Same cross-sectional view as Fig. 4(b). (b) Intensity profile corresponding to the line shown in Fig. 5(a).

Fig. 6 (a) Same cross-sectional view as Fig. 4(b). (b) Intensity profiles corresponding to the white thin dotted line including the $\mathrm{K} 8$ fiber and the white thick dotted line including the F830 fiber in Fig. 6(a). (c) Enlarged intensity profile for the F830 single fiber in Fig. 6(b). (d) Enlarged intensity profile for the K8 single fiber in Fig. 6(b). 


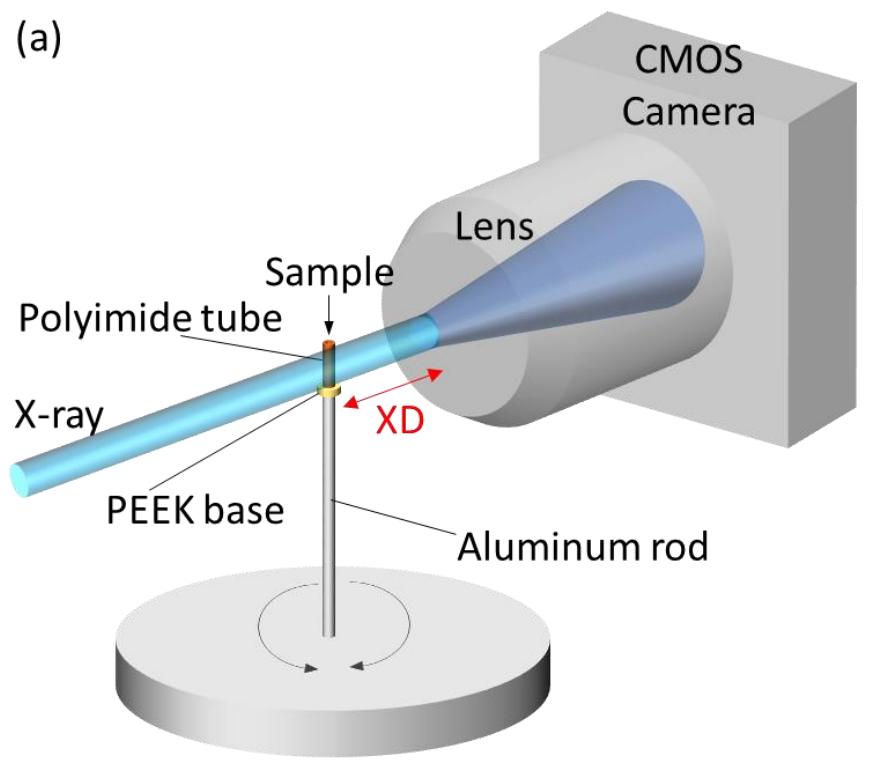

(b) Sample

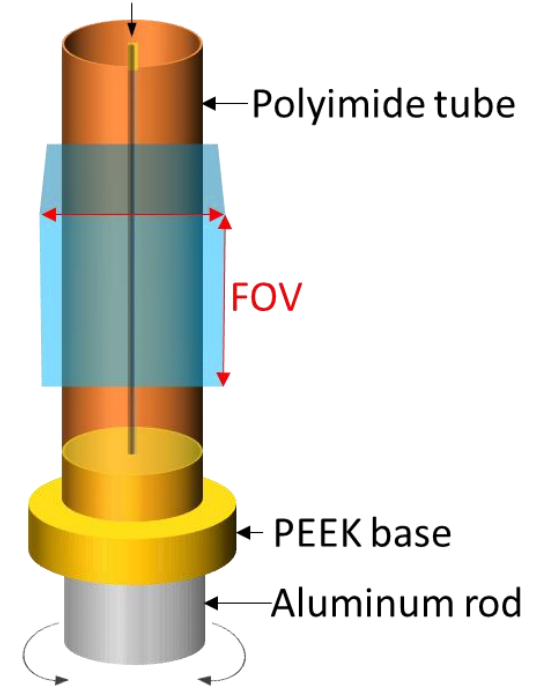

Fig. 1 

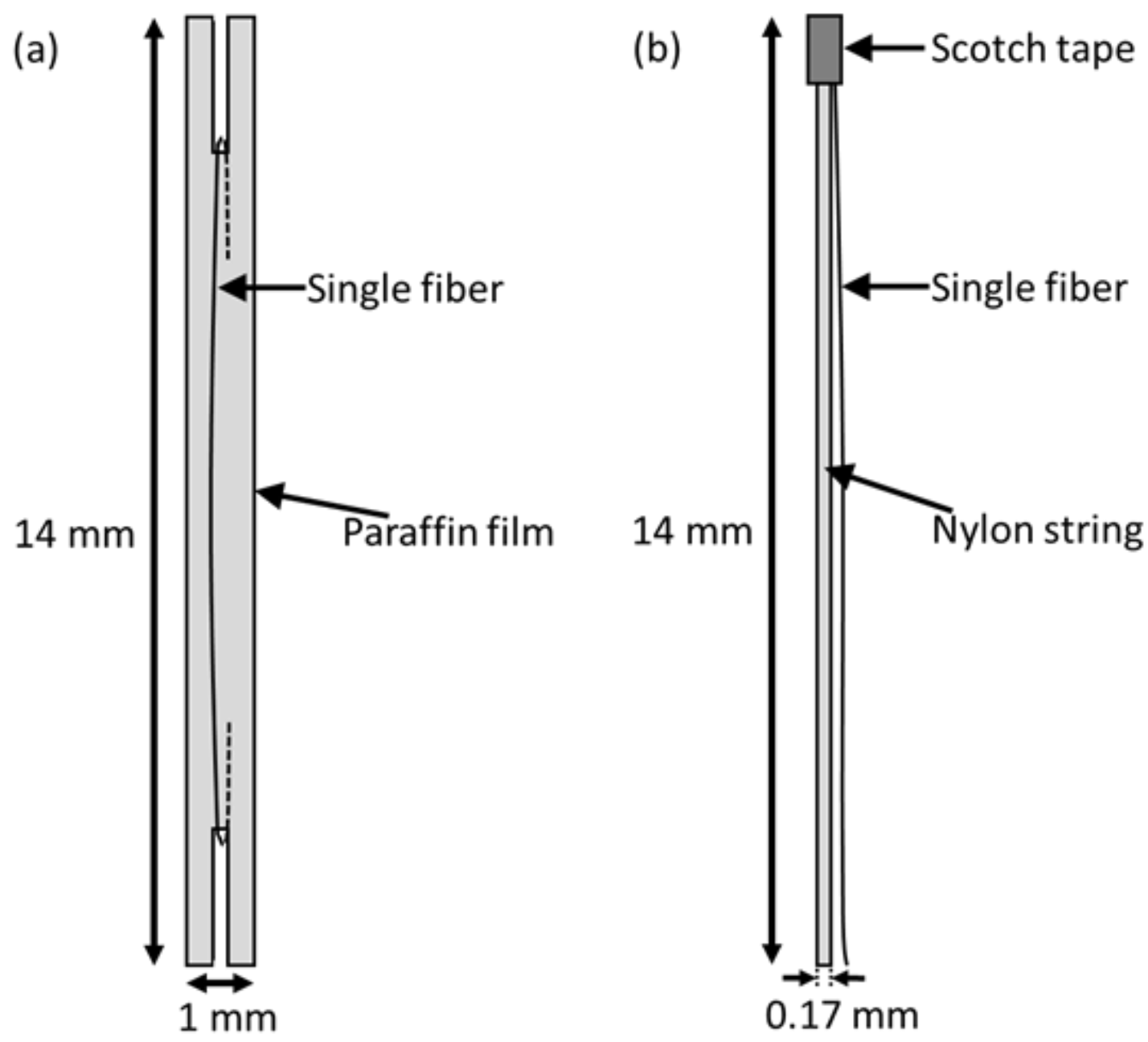

Fig. 2 


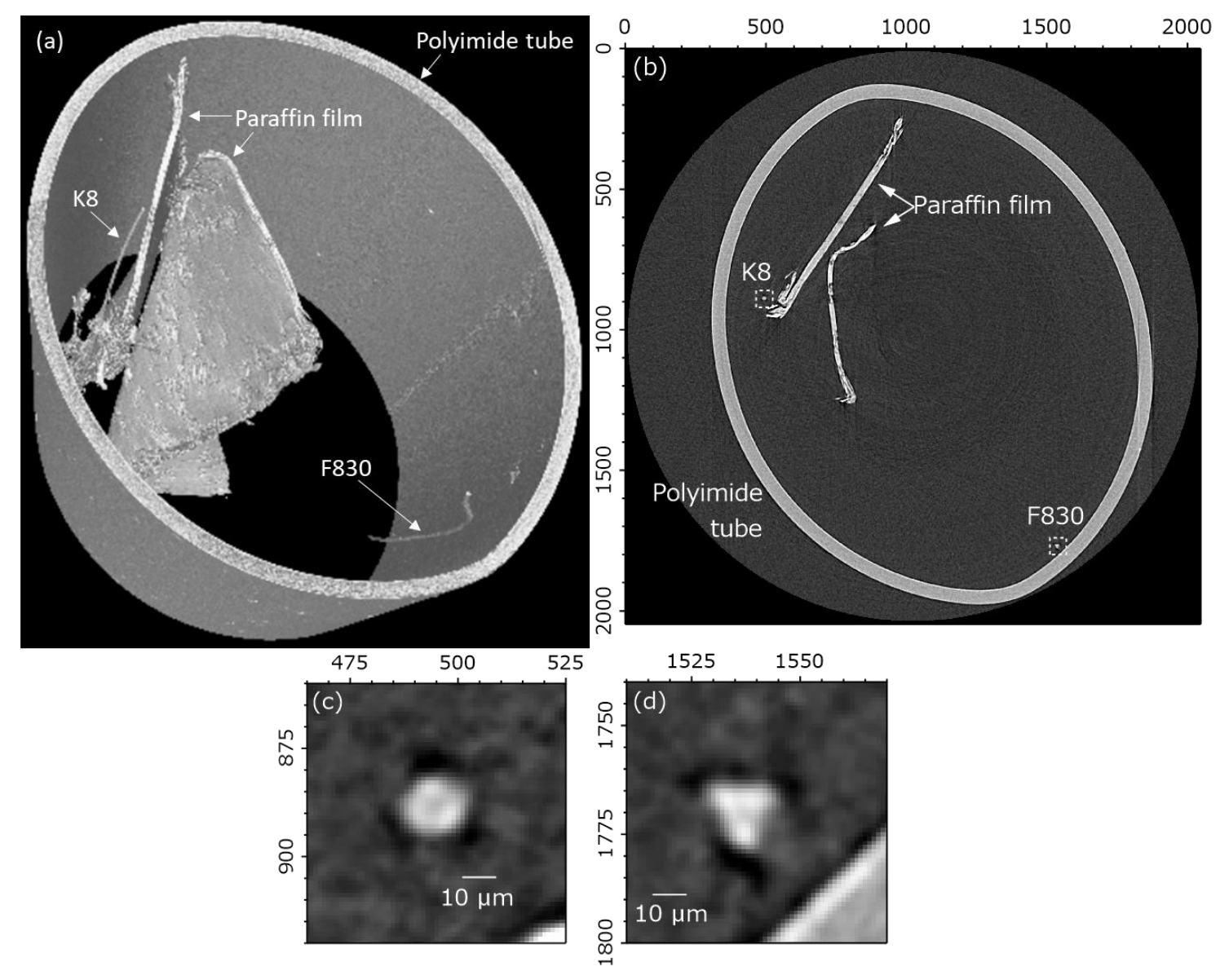

Fig. 3 


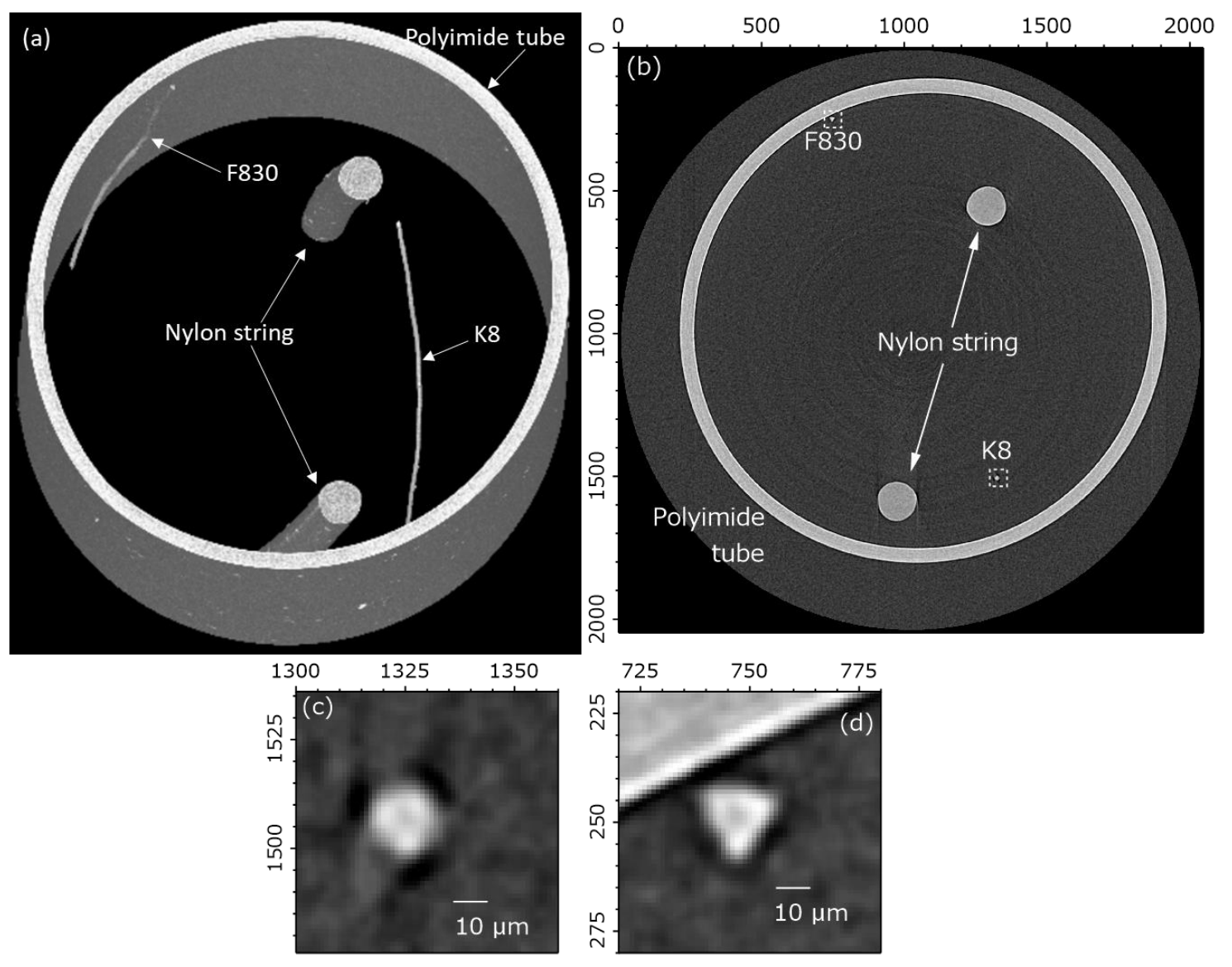

Fig. 4 


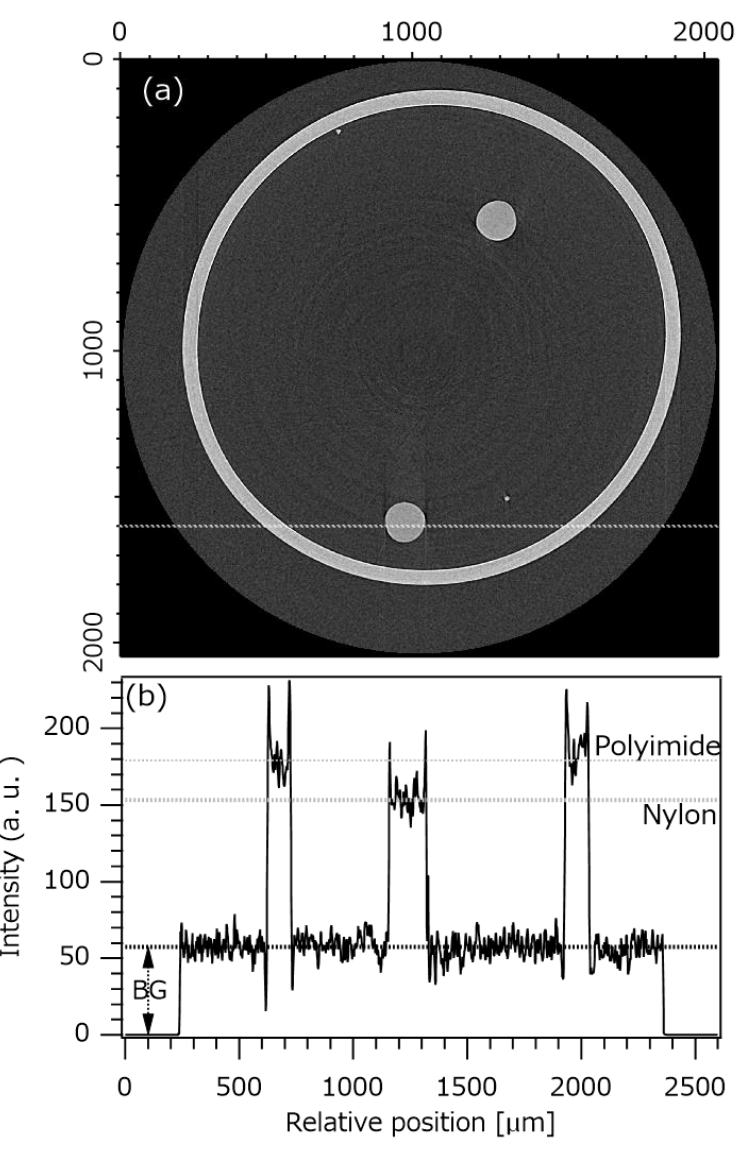

Fig. 5 

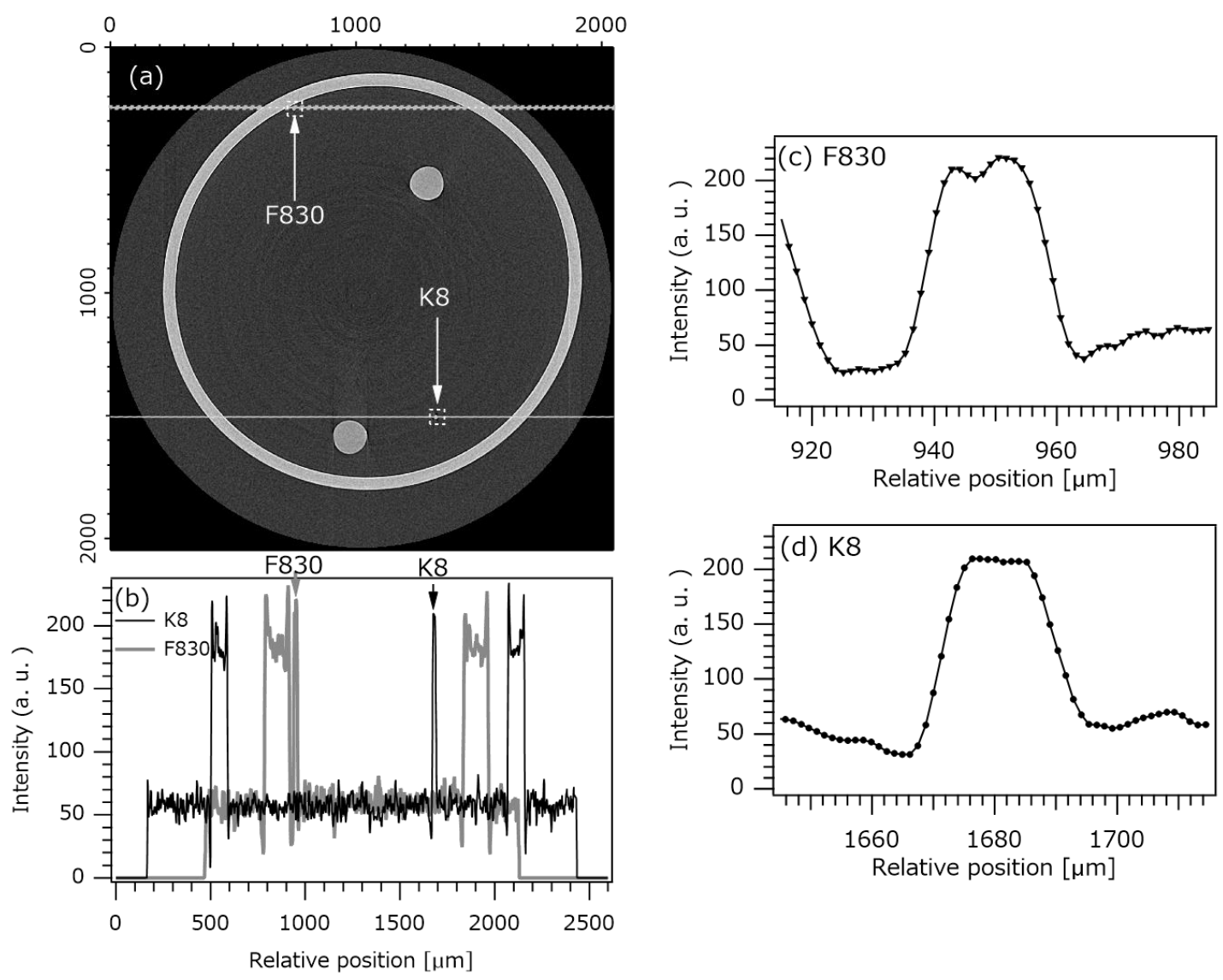

Fig. 6 
Graphical Index

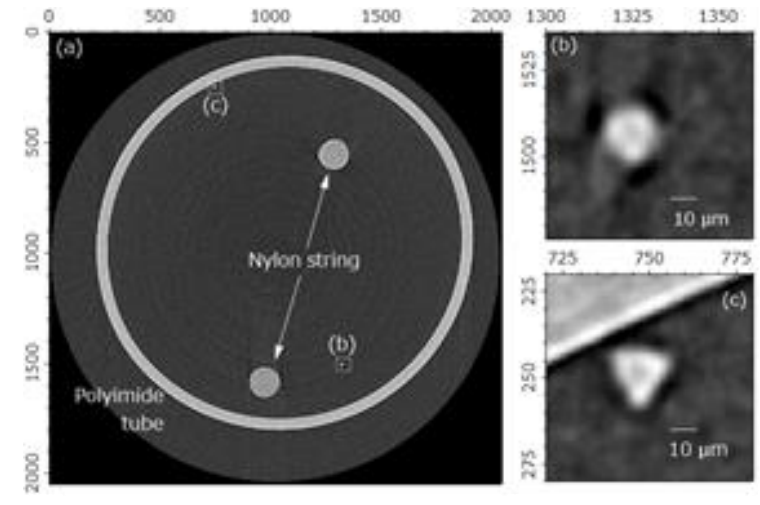

